\title{
A field trial to assess the environmental biosafety of codA-transgenic Eucalyptus camaldulensis cultivation
}

\author{
Xiang Yu ${ }^{1, \dagger, a}$, Akira Kikuchi ${ }^{1, *, \dagger}$, Etsuko Matsunaga ${ }^{2}$, Akiyoshi Kawaoka $^{2}$, \\ Hiroyasu Ebinuma ${ }^{2, b}$, Kazuo N. Watanabe \\ ${ }^{1}$ Faculty of Life and Environmental Sciences, Gene Research Center, University of Tsukuba, Tsukuba, Ibaraki 305-8752, Japan; \\ ${ }^{2}$ Agri-Biotechnology Research Laboratory, Nippon Paper Industries Co. Ltd., Tokyo 114-0002, Japan \\ *E-mail: kikuike@gene.tsukuba.ac.jp Tel: +81-29-853-7743 Fax: +81-29-853-7729
}

Received November 13, 2012; accepted April 30, 2013 (Edited by Y. Ozeki)

\begin{abstract}
The first Japanese field trial testing the effects of genetically modified (GM) forest trees was conducted over a period of 4 years. Salt tolerance was conferred on Eucalyptus camaldulensis by introducing the soil microbe-derived choline oxidase $(\operatorname{cod} A)$ gene into this plant. Three individual lines were planted in an isolated field owned by the Gene Research Center at the University of Tsukuba, following approval for type I use by the Ministry of Education, Culture, Sports, Science and Technology, Japan, and the Japanese Ministry of the Environment. Soil populations of fungi, actinomycetes, and bacteria fluctuated during sampling, but any differences introduced were observed only transiently, and no long-term change in the population was observed during the trial. Both the soil-mix as well as the sandwich method was employed to evaluate allelopathic activity of codA-transgenic E. camaldulensis. By the soil mix method, both GM and non GM-derived plant samples were found to exert negative allelopathic effects on seed germination, with an annual fluctuation. Overall, no significant differences were observed between the transformant lines and their bulk seed-derived non-transformants. The sandwich method also revealed an annual fluctuation. A comparison of the non-transformants and transformants revealed no significant differences in their allelopathic activity. Considering the practical use of these transgenic E. camaldulensis, they will no doubt be planted in some salinized sites. Thus, evaluating their impact on the ecosystem in saline conditioned fields might be important in the future.
\end{abstract}

Key words: $\operatorname{codA}$, environmental biosafety, Eucalyptus, field trial, salt tolerance.

The development of genetic engineering technology in the past 3 decades has enabled scientists to introduce traits found in other species directly into plants. Genetic engineering has contributed to various basic studies, has helped clarify gene function or the mechanism underlying various physiological phenomena, and has been utilized in applied sciences to improve plant performance. Genetic modification has been used to develop herbicide tolerance or biotic stress tolerance (Clive 2011; FAO 2004; Kikuchi et al. 2008) in crops. However, assessing the environmental biosafety of each genetically modified (GM) plant is essential prior to practical use. Precautionary procedures for systematic and case-by-case evaluations of transfer of GM plants from container to field conditions are defined by the Cartagena Protocol on Biosafety (CPB; Schomberg 1998).

A long-term environmental vision for utilizing the natural resources of the earth is necessary to establish sustainable development. The strategy of generating abiotic stress-tolerant plants, particularly perennial species, is one approach to achieve such a goal. Classic breeding is relatively efficient at conferring resistance to biotic stresses (that arise from exposure to plant host-specific insects or diseases), but appears to be weak at conferring tolerance to abiotic stresses. Genetic engineering techniques are an excellent way to improve abiotic stress tolerance in plants, particularly in tree species. The development of abiotic stress-tolerant trees may be helpful in combating global warming, by the expansion of the new planting area. Additionally, these trees might not compete with crops grown for the purpose of food production.

We have collaborated for 15 years to develop GM Eucalyptus lines that exhibit tolerance to abiotic

Abbreviations: codA, choline oxidase; CPB, Cartagena Protocol on Biosafety; EC, electrical conductivity; GM, genetically modified; PTYG, peptone, tryptone, yeast extract, and glucose; MEXT, Ministry of Education, Culture, Sports, Science and Technology; TERC, Terrestrial Environment Research Center

${ }^{\dagger}$ These authors contributed equally to this work.

${ }^{\text {a }}$ Present address: RIKEN Center for Sustainable Resource Science, Yokohama, Japan.

${ }^{b}$ Present address: Faculty of Textile Science and Technology, Shinshu University, Ueda, Japan.

This article can be found at http://www.jspcmb.jp/

Published online September 5, 2013 
stress. We initiated a performance evaluation and environmental biosafety assessment of 3 different Eucalyptus camaldulensis lines (12-5B, 12-5C, and 20-C) containing the Arthrobacter globiformis choline oxidase ( $\operatorname{codA}$ ) gene (Ikuta et al. 1977), under semi-containment. The growth profile and morphological features of these transformants did not differ from those of their nontransformed counterparts (Kikuchi et al. 2008). These transformants survived a single treatment of $2 \mathrm{M} \mathrm{NaCl}$ (Kikuchi et al. 2006), and the impact of both types (transformant and non-transformant) of lines on the local soil microbe population was similar. Furthermore, no significant difference in allelopathic activity of leaves was observed (Kikuchi et al. 2006, 2009).

Based on these results, 3 applications for individual field trials (type I use in Japan) were pre-submitted to the Ministry of Education, Culture, Sports, Science and Technology (MEXT) in Japan. Although various GM trees lines have been developed, only a small proportion of these have been tested by field trials, with only 25 such tests having been conducted worldwide since 2004 (FAO 2004). In Japan, only a single field trial (which tested a GM ornamental fruit tree) had been performed thus far (FAO 2004). After 1.5 years, final applications for type I use were submitted to MEXT and the Japanese Ministry of the Environment on March 16, 2005. This is first type I use submission to MEXT, the requirement for which was established on February 19, 2004, after enforcement of a CPB-related act. These field trials were approved on October 12, 2005 (J-BCH 2005a). The trial duration was up to 5 years from the date of approval. To prevent dispersal of transgenic biomaterial (plants and pollen) from the trial field, various measures such as cutting of flower buds and covering the trial field with a net were included as necessary procedures in the study protocol.

During the general cultivation of herbal crops, almost crops except rice are not planted in the same field due to use of the rotation system. Other than canola, almost all crops have difficulties surviving without agricultural management. Successive environmental biosafety assessments may not be emphasized for the above reasons. However, several perennial plants species were planted, after stating that the plants would be maintained in the same location for several years. For this reason, environmental biosafety assessments should be continuously performed prior to planting of perennial GM plants. In this study, the first field trial of a GM species of forest trees was performed in Japan (FAO 2004; J-BCH 2004, 2005b), with a 4-year continuous environmental biosafety assessment examining the allelopathic activity of the GM tree and changes in the local soil microbe composition.

\section{Materials and methods}

\section{Plant materials}

The expression of the $\operatorname{cod} A$ gene in transgenic plants is driven by the CaMV 35S constitutive promoter (Kikuchi et al. 2009). The transgenic and non-transgenic plantlets were propagated by tissue culture. All propagated plantlets were grown for 3 months in a quadrangular pyramid pot $\left(12.5 \mathrm{~cm}\right.$ high $\left.\times 4.5 \mathrm{~cm}^{2}\right)$ placed in a special netted-house (Yu et al. 2009). The bulk seeds of non-transgenic E. camaldulensis were planted and cultivated for 6-9 weeks in a special netted-house. The soil used was a mixture of pumice, red clay, and trass $(1: 1: 1)$, and is termed Kanuma soil. Small saplings $(10-20 \mathrm{~cm}$ in height) were planted in the trial field.

\section{Field trial conditions}

The field trial was carried out from October 2005 to November 2009 in an isolated field owned by the Gene Research Center at the University of Tsukuba. A clay layer exists about $70 \mathrm{~cm}$ below the ground in this field. To avoid the influences from the soil microbe community in the adjacent field, bulk seedderived, non-transgenic E. camaldulensis trees were planted around the experimental trees to form a buffer zone ( $S$ in Figure 1A). Small saplings were planted at 1-m intervals in the trial field $(7 \mathrm{~m} \times 8 \mathrm{~m}$; Figure $1 \mathrm{~A}, \mathrm{~B})$. Various measures were undertaken to protect small saplings from the cold; the trial field was covered with a plastic greenhouse (Figure 1C) from October 2005 to May 2006, and each small sapling was covered by a plastic dome $(\phi 30 \mathrm{~cm}$; Figure 1D) during the first winter (December 2005-March 2006). This field trial was performed with the approval of MEXT and the Japanese Ministry of the Environment for type I use (05-26P-0001, -0002, and -0003).

Just before an expected rainfall, the trial field was treated with $2.0 \mathrm{~kg} \mathrm{~m}^{-2}$ or $2.5 \mathrm{~kg} \mathrm{~m}^{-2}$ salt on August 7 , and on September 18, 2009, respectively. The electrical conductivity (EC) observed at an approximate depth of $5 \mathrm{~cm}$ was monitored from August 7 to November 24 at 12 points in the trial field (yellow stars in Figure 1A). The vertical EC distribution was monitored at depth between 5 to $50 \mathrm{~cm}$, at 4 points, on October 14, 2009 (green stars in Figure 1A). The EC at each point was measured 3 times a day with an EC meter (FieldScout ECTestr 11+; Spectrum Technologies, Inc., Plainfield, IL). The average of these readings was considered the daily EC (Figure 2). The rainfall data were obtained from the Terrestrial Environment Research Center (TERC) at the University of Tsukuba (Figure 2). The distance between TERC and the trial field was about $500 \mathrm{~m}$.

\section{Examination of the microbial population of the rhizosphere by the plate culture method}

Soil was sampled from each spot in the trial field cultivated with the transgenic E. camaldulensis without bulk seeds nontransformant. The sampling points were located $20 \mathrm{~cm}$ away from the tree. After removing the surface soil, the field soil was collected from the rhizosphere, at a depth of $5-15 \mathrm{~cm}$ 

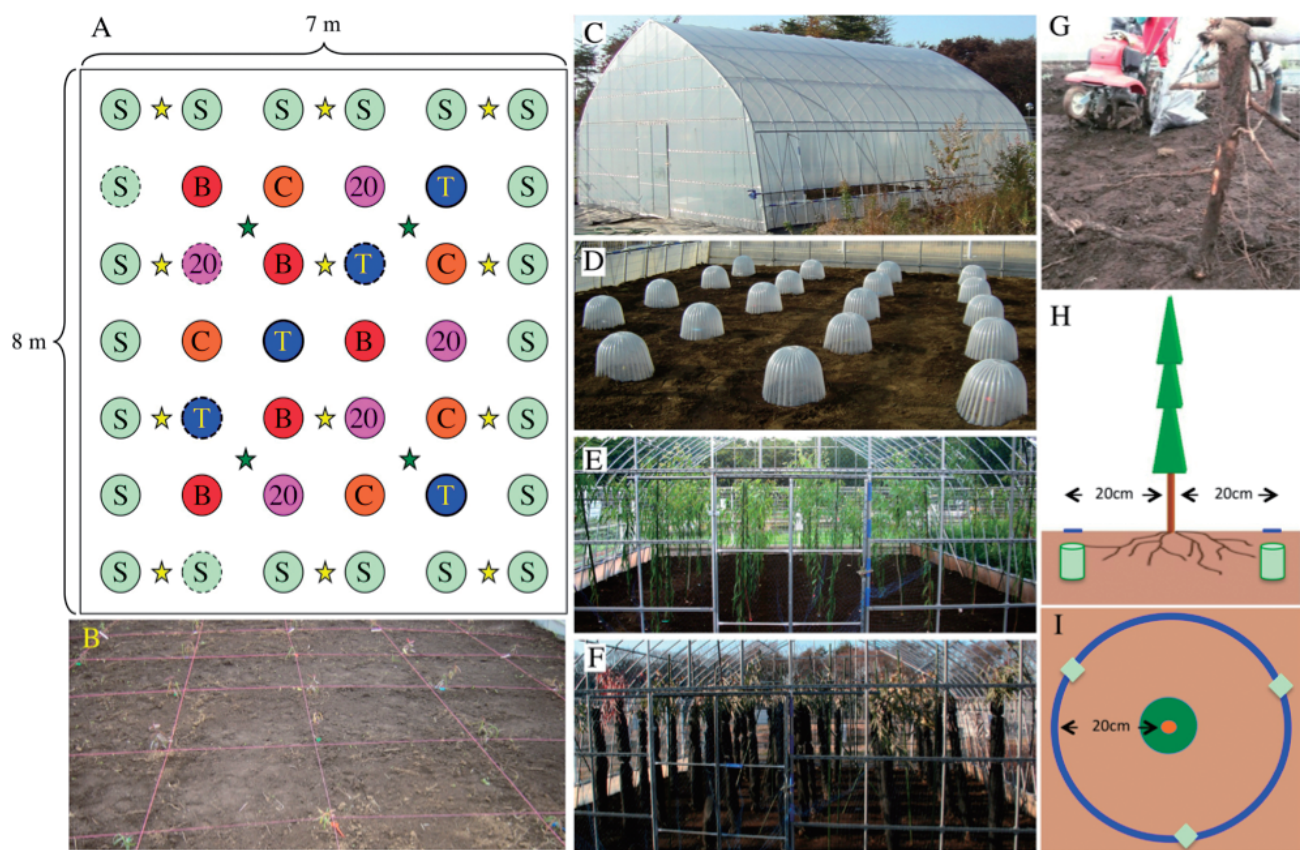

Figure 1. Field trial of salt-tolerant transgenic Eucalyptus camaldulensis plants. The field trial was performed in an isolated field owned by the Gene Research Center at the University of Tsukuba, from October 2005 to November 2009. It was a field designed specifically for this trial (A). B, C, and 20 indicate the location of individual transformants, $12-5 \mathrm{~B}, 12-5 \mathrm{C}$, and $20-\mathrm{C}$, respectively. T indicates the non-transformant plant, T-5. S indicates the non-transformant plants derived from bulk seeds. Dotted circles indicate the trees that died during winter. Yellow stars indicate the points where the EC was monitored at the surface (at a depth of $5 \mathrm{~cm}$ ). Green stars indicate the points where the vertical distribution of the EC was monitored, at depths between 5 and $50-\mathrm{cm}$. An image of the trial field taken immediately after planting is shown (B). The small saplings were planted at 1-m intervals in the trial field. The trial field was covered with a plastic greenhouse to protect small saplings from the cold (C). An image of the planted saplings covered with a plastic dome is shown (D). An image of the tree growth during the first summer is shown (E). After removing the plastic sheet from the house, a frame was used for covering the trial field with net. An image of the planted tree growth during the first winter (F). Plants were protected from frost and cold from December to May every year. All trees were dug to their root zone after the field trial. As a result, no tree had roots going into the clay layer $(\mathrm{G})$. A depiction of the area $(20 \mathrm{~cm}$ from the tree) from where the soil samples were collected is shown (H). A depiction of the location of the 3 points around a single tree at which field soil collection (at a depth between 5 to $15 \mathrm{~cm}$ ) occurred is shown (I).
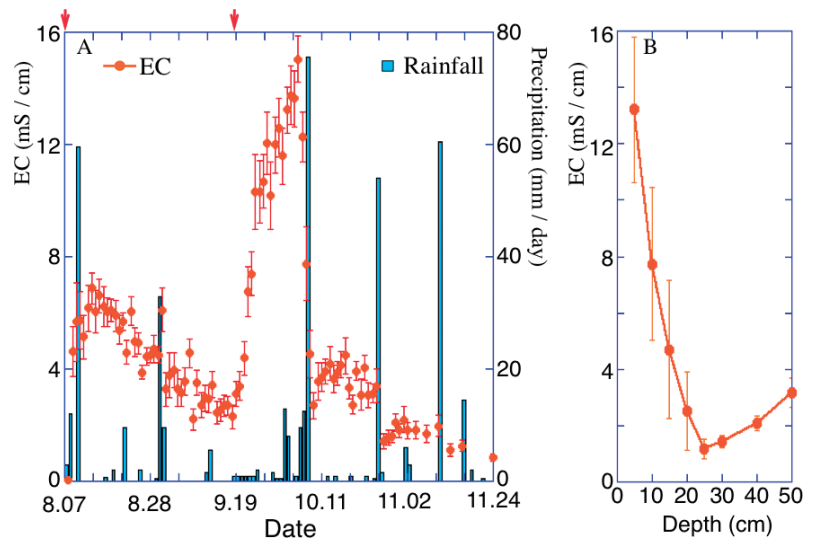

Figure 2. Alterations in the electrical conductivity (EC) in the trial field. The EC was monitored during salt treatment. Daily changes were monitored at the surface (at a depth of $5 \mathrm{~cm}$ ) at 12 points in the trial field (Figure 1A). Red arrows indicate the day the trial field was treated with salt. The average value of the EC at all points examined per day is displayed (A). Vertical EC distribution was examined at 4 points, on a single day (October 14, 2009), along a 5-50 depth. The average value of all points is shown (B). Most of the salt remained on the surface. The EC indicated is the average of the monitored points. The error bars indicate standard error of mean.
(Figure $1 \mathrm{H}$ ). Half of each sample was dried at $80^{\circ} \mathrm{C}$ to obtain dry weight, and the remaining half of the sample was used to study the microbial population. This evaluation was performed using a previously reported method (Shiomi et al. 1992; Yu et al. 2013). Fungal growth was monitored in an oxytetracyclineglucose-yeast extract agar, by incubating the culture plates for 3 days at room temperature. The growth of actinomycetes and other bacteria was monitored by culture on peptone, tryptone, yeast extract, and glucose (PTYG) agar for 7 days at room temperature. After counting the total number of colonies on growing on PTYG agar, the bacterial colonies were scraped with a glass spreader with $70 \%$ ethanol. The remaining colonies on the plate were counted as actinomycetes. Soil samples were taken from 5 individual trees per line, and each soil sample was tested in triplicate.

\section{The soil-mix method}

The soil-mix method, commonly used in biosafety assessments, is used to assay the allelopathic activity of soil leachates released from dried leaves. This method examines the effects of leachates. It is intended to mimic natural conditions wherein plant matter from senescing leaves and other parts of the plant enters the soil, and is then bound to soil particles. Five 
lettuce seeds (Lactuca sativa L. var. capitata L.: cv. Great Lakes 366; Takii Seed, Kyoto, Japan) were placed on the surface of a leaf-soil mixture in each cell (Yu et al. 2013). Germination rate was recorded after a 5 -day incubation at $25^{\circ} \mathrm{C}$ in the dark. Five individual trees from each line were tested.

\section{The sandwich method}

The sandwich method is another method to assay the allelopathic activity of leachates released from dried leaves (Fujii et al. 2003, 2004). By monitoring the growth of lettuce seeds on agar medium, this method has been used to evaluate the allelopathic activity of codA-transgenic Eucalyptus (Kikuchi et al. 2006, 2009). In this study, the assay was performed following a previously reported method (Fujii et al. 2003; Yu et al. 2013). Allelopathic activity was evaluated by measuring the length of radicles and hypocotyls of lettuce seeds (5 seeds per sample replicate). Data from the longest and shortest seedling in each well were excluded. Nine wells were prepared for each evaluation, and samples from five individual trees of each genotype were tested. The results were presented as growth relative to that observed in a control (lettuce seeds grown on agar in the absence of added leaf leachates) group.

\section{Results and discussion}

\section{A field trial of codA-transgenic Eucalyptus trees}

E. camaldulensis can be grown in a range of habitats, from tropical to subtropical, as well as in warm or temperate areas. Its growth is weak under cold conditions (Duke 1983). A previous study examining $E$. camaldulensis plantation in the same field revealed that half of the plants could not be overwintered without protection (Kikuchi et al. 2006). In this trial, small saplings were planted in an isolated field at the end of October 2005. As the planted saplings were still small $(<20 \mathrm{~cm})$ in early December 2005 , it was easy to protect them from frost and cold by using a plastic greenhouse and dome (Figure 1C,D). All saplings survived to spring 2006. This type I use required a containment of the transgenic biological material, and hence, plantation and cultivation were carried out under a net, or some other type of cover (Figure 1E). Branches were promptly cut to keep the plants entirely under the net, and all tree trunks were cut to a height of $150 \mathrm{~cm}$ every autumn. Frost and cold protection was provided from December to May every year (Figure 1F). Despite this protection, some trees did not survive until the final year, due to a failure to overwinter (dotted circle in Figure 1A).

The field was treated twice $\left(2.0 \mathrm{~kg} \mathrm{~m}^{-2}\right.$ and $\left.2.5 \mathrm{~kg} \mathrm{~m}^{-2}\right)$ with salt during the trial. The average EC and daily rainfall are shown in Figure 2A. The EC decreased after heavy rainfalls. Surface weeds completely withered, but all E. camaldulensis trees survived with some damage to the leaves. After exposing roots of the trees, we discovered that almost all of them had reached the surface of the clay layer, but they did not extend into this layer (Figure 1G). We examined the horizontal distribution of EC at 4 points by digging a hole (indicated by a green star in Figure 1A). The EC at the surface (up to $5 \mathrm{~cm}$ deep) was $>12$ but was $<4$ in layers deeper than $20 \mathrm{~cm}$ (Figure 2B). Salt tolerance of young trees was evaluated by treating the soil with a $200 \mathrm{mM} \mathrm{NaCl}$ solution (EC 11) (Lelmen et al. 2010). The EC values at depths above $20 \mathrm{~cm}$ varied widely at the different points examined. In particular, at a depth of $15 \mathrm{~cm}$, the values ranged from 0.34 to 11.70 . The variations may be attributable to a water vein in the field. For this reason, field conditions during this field trial may have been insufficient to evaluate the salt tolerance of the transformants.

An environmental biosafety assessment examining the effect of codA-transgenic Eucalyptus on soil microbes

Alterations in soil microbes were monitored for 4 years (Figure 3). The populations of fungi, actinomycetes, and other bacteria fluctuated during sampling. In particular, during the first year after planting, the fluctuation in the bacterial population was remarkable (Figure 3C). A significant difference between a non-transformed (T-5) line and some of its transgenic counterparts was detected at some samplings, but these differences appeared transient, and no long-term changes were observed during the trial. Overall, the impact of transgenic E. camaldulensis on the soil microbe community did not significantly differ from that of the non-transformants.

On the other hand, the soil densities of fungi and bacteria increased after salt treatment (Figure 3). Although no differences in this parameter were observed between the non-transformant and transformant lines, the increase in soil bacteria in both groups was remarkable (Figure 3C). To examine the relationship of the microbial community with salt treatment, further monitoring of bacteria in salt-treated soil without trees may be necessary.

\section{Allelopathic activity of codA-transgenic Eucalyptus}

The soil-mix method was applied in combination with the sandwich method to evaluate allelopathic activity of $\operatorname{codA}$-transgenic E. camaldulensis at the end of October each year, except in 2009, when it was carried out in November. Allelopathic activity of biomaterial that had leached from leaves was evaluated. Five bulk seedderived trees from the buffer zone ( $\mathrm{S}$ in Figure 1A) were selected to provide non-transformant sample materials. Eucalyptus leaves contain various allelopathic molecules, including 1,8-cyneole (Khan et al. 2008). When evaluated by the soil-mix method, samples from all groups exerted negative effects on seed germination, with annual 


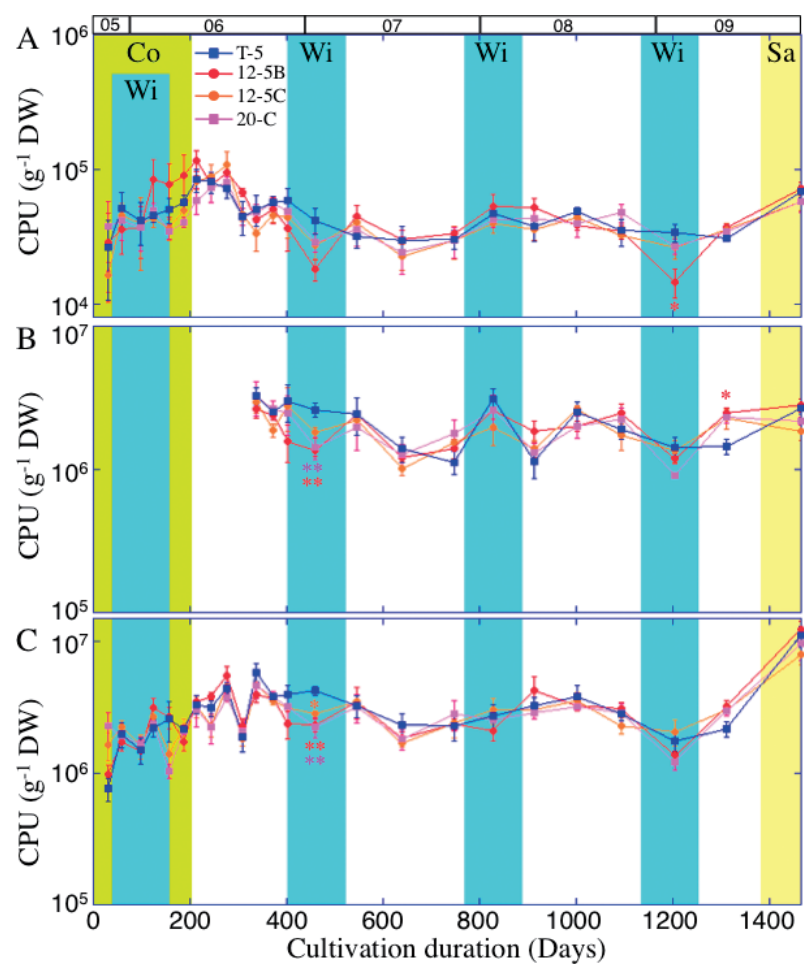

Figure 3. Changes in the soil microbe population in the trial field. Alterations in fungi (A), actinomycetes (B), and other bacteria (C) were monitored for 4 years in the soil in which the transgenic lines was planted. Five individual trees from each line were used. The green background denotes the period when the trial field was covered with a plastic greenhouse (Co). The pale-blue background indicates the winter season from December to March (Wi). The yellow background indicates the duration after salt treatment $(\mathrm{Sa})$. The numbers on the uppermost panel in the graph indicate years. Error bars indicate the standard error of mean. Statistical differences among the lines were evaluated by ANOVA, and then by the Tukey-Kramer method. Asterisks indicate a significant difference between T-5 and each transformant, obtained by evaluation by the Tukey-Kramer method $(* p<0.05, * * p<0.01)$.

fluctuations. However, the differences between the activity of the various lines did not reach significance any of the years studied (Figure 4).

Annual fluctuations also observed with the sandwich method. All samples (collected from both transformants and non-transformants) inhibited radicle and hypocotyl growth when the lettuce seeds were treated with $50 \mathrm{mg}$ of the leaf leachate (Figure 5A,B). However, some minor differences were observed in when the seeds were treated with $10 \mathrm{mg}$ leaf leachate; all samples, excluding those from T-5 (in 2006 and 2007) and bulk seed derived trees (in 2007) inhibited radicle growth (Figure 5C). In contrast, all samples (other than the 12 -5C-derived sample in 2009) increased hypocotyl growth (Figure $5 \mathrm{D})$. Before the salt treatment, there were no significant differences observed between the non-transformant and transformant groups. After the salt treatment (in 2009), the effect of the T-5 line-derived samples differed significantly from that of other lines tested (Figure 5B,

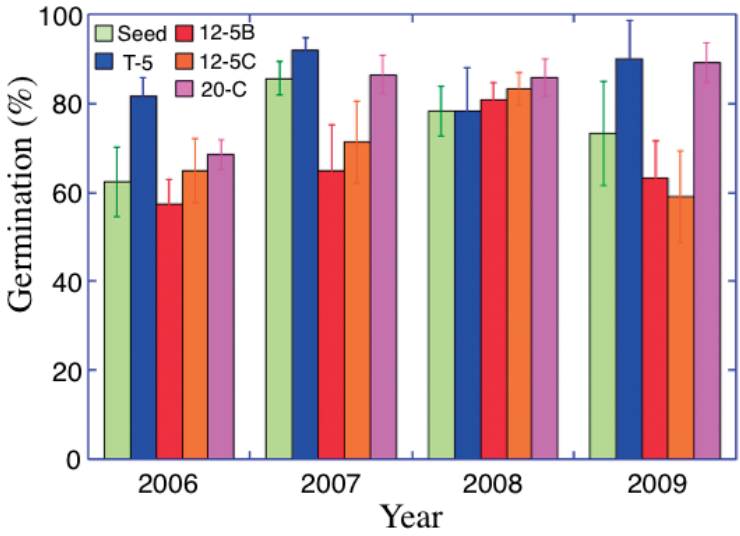

Figure 4. Results of allelopathy testing of transgenic and nontransgenic leaf leachates by the soil-mix method. Light-green bars represent non-transformants derived from bulk seeds (Seed). Blue bars represent a non-transformant, T-5. Red, orange, and pink bars represent the 12-5B, 12-5C, and 20-C transformants, respectively. Error bars indicate standard error of mean. Five individual trees per genotype and six replicates per individual tree were tested. There were no significant differences observed between any of the lines (by ANOVA) in all the years of study.
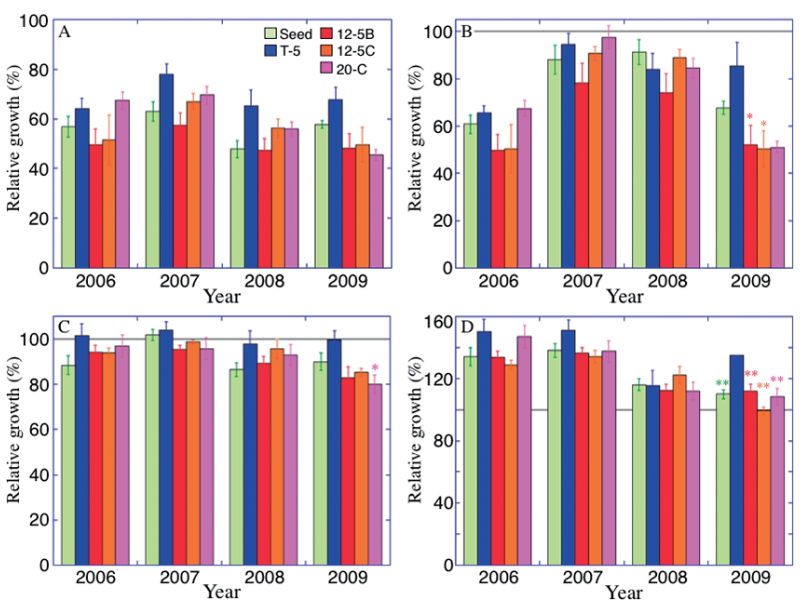

Figure 5. Results of the allelopathy testing of transgenic and nontransgenic leaf leachates using the sandwich method. The effects of 2 quantities (50 and $10 \mathrm{mg}$ ) of dry leaves were examined. A, Radicle growth stimulated by $50 \mathrm{mg}$ of dry leaves; B, Hypocotyl growth stimulated by $50 \mathrm{mg}$ of dry leaves; C, Radicle growth stimulated by $10 \mathrm{mg}$ of dry leaves; D, Hypocotyl growth stimulated by $10 \mathrm{mg}$ of dry leaves. Light-green bars represent non-transformant bulk seedgerminated trees (Seed). Blue bars represent a non-transformant, T-5. Red, orange, and pink bars represent the 12-5B, 12-5C, and 20-C transformants, respectively. Error bars indicate standard error of mean. Five individual trees per genotype and 9 replicates per individual trees were tested for both (50 or $10 \mathrm{mg}$ ) leaf treatments. Statistical differences among lines were evaluated by ANOVA. Significant differences between certain groups were observed in growth of hypocotyls following treatment with $50 \mathrm{mg}(\mathrm{B})$ and $10 \mathrm{mg}$ (D) of dry leaves, or in the growth of radicles (C) following treatment with $10 \mathrm{mg}$ of dry leaves. Differences between groups were also evaluated by the Tukey-Kramer method. No significant differences were observed between the effects exerted by non-transformant bulk-seed germinated trees and each transformant line. Asterisks denote a significant difference between the indicated line and T- 5 line, obtained by evaluation by the Tukey-Kramer method $(* p<0.05, * * p<0.01)$. 
$\mathrm{C}$, and $\mathrm{D}$ ), except when examining radicle growth in the $50 \mathrm{mg}$ leachate group (Figure 5A). The T-5 line-derived leaf leachates tended to show the lowest allelopathic activity; a significant difference in the hypocotyl growth was observed with comparing the effect of T-5 to that of the bulk seed-derived leachates, in the $10 \mathrm{mg}$ leaf treatment group (Figure 5D). In previous evaluation of those tranegenic lines, line T-5 did not use as nontrasfrormant line (Kikuchi et al. 2006, 2009), it might be the nature of line T-5. In this study, allelopathic activity of the transformant lines did not differ from the bulkseed derived non-transformants growing in the buffer zone. Overall, the allelopathic activity observed in our study showed no clear trend (of either an increase or a decrease), and the allelopathic activity of the transformants was in the same range as that of the nontransformants during the 4-year observation period. As this is a perennial species, a long-term environmental biosafety assessment is essential to evaluate these transgenic trees.

\section{The importance of risk assessment for transgenic plants in a field trial}

This is the first perennial monitoring trial conducted under field conditions in Japan. Because this species is perennial, a long-term monitoring of its environmental biosafety following genetic modification is important. A previous study, carried out in a special netted-house (a condition of semi-containment), examined the effect of genetic modification on the allelopathic activity or the soil microbe impact, and revealed no significant differences between these transgenic lines and a nontransgenic line (Kikuchi et al. 2006, 2009). Although significant differences were observed at certain times in the years of study, these effects were not long-lived. Moreover, the impact on the soil microbe community of the transformants exhibited no significant positive or negative trend (Figure 3). Based on the present field evaluation, codA-transgenic E. camaldulensis and its nontransformed counterparts may have the same impact on the soil microbe community. Perennial monitoring in a field trial is useful in evaluating alterations in an ecosystem, the impacts of a physiological change, and the effects of seasonal variation on transgenic plants. The 2 evaluation methods revealed no significant differences in the allelopathic capacity following genetic modification (Figures 4, 5). The environmental impact of these transformants did not change based on cultivation either in the field or in a special netted-house.

Fungal and bacterial populations in the local soil surrounding all the lines increased after salt treatment (Figure 3). As the effect of salt treatment was not evaluated during cultivation in the special nettedhouse, and only a single sample was collected after salt treatment in this study, the meaning and significance of these alterations need to be further examined. The transgenic E. camaldulensis trees will be planted at other salinized sites, and evaluating their impact on the ecosystem following cultivation in saline-conditioned fields in the future will be useful. Because of Japanese regulations, it is necessary to perform the environmental biosafety assessment under semi-containment, in a special netted-house followed by a field trial in an isolated field, before proceeding to practical planting. This study revealed seasonal fluctuations and alterations in the soil microbiota after salt treatment (Figure 3). The precautionary repetitive method used in this study is a rational approach to evaluate a transformant. It will be necessary to conduct a field trial evaluating adult trees grown in soil treated with salt for several years, to develop a practical cultivation method for these salttolerant transgenic E. camaldulensis trees.

\section{Acknowledgements}

We thank Dr. Norio Murata for providing the $\operatorname{cod} A$ gene. This study was supported in part by the Japan Society for the Promotion of Science (JSPS; Grant-in-Aid for Scientific Research (A) No. 17208001 and 21248001) and by the Gene Research Center, University of Tsukuba. The authors are very grateful for their support.

\section{References}

Clive J (2011) Global Status of Commercialized Biotech/GM Crops: 2011. ISAAA Brief No.43. ISAAA: Ithaca, NY

Duke AJ (1983) Eucalyptus camaldulensis Schlecht. In Handbook of Energy Crops. unpublished. http://www.hort.purdue.edu/ newcrop/duke_energy/Eucalyptus_camaldulensis.html

FAO (2004) Preliminary review of biotechnology in forestry, including genetic modification. Forest Genetic Resources Working Paper 59. Forest Resources Development Service Working paper FGR/59E, Forest Resources Division, FAO, Rome, Italy

Fujii Y, Parez SS, Parvez MM, Ohmae Y, Iida O (2003) Screening of 239 medicinal plant species for allelopathic activity using the sandwich method. Weed Biol Manage 3: 233-241

Fujii Y, Shibuya T, Nakatani K, Itani T, Hiradate S, Parvez MM (2004) Assessment method for allelopathic effect from leaf litter leachates. Weed Biol Manage 4: 19-23

Ikuta S, Imamura S, Misaki H, Horiuchi Y (1977) Purification and characterization of choline oxidase from Arthrobacter globiformis. J Biochem 82: 1741-1749

Japan Biosafety Clearing-House (J-BCH) (2004) http://www.bch. biodic.go.jp/english/lmo_2004.html

Japan Biosafety Clearing-House (J-BCH) (2005a) Eucalyptus tree containing salt tolerance inducing gene $\operatorname{cod} A$ derived from Arthrobacter globformis (codA, Eucalyptus camaldulensis Dehnh.) (12-5B, 12-5C and 20-C) 05-26P-0001, -0002, and -0003. 2005-10-12

Japan Biosafety Clearing-House (J-BCH) (2005b) http://www.bch. biodic.go.jp/english/lmo_2005.html

Japan Biosafety Clearing-House (J-BCH) (2007) High cellulose rich white poplar (trg300-1, trg300-2) (AaXEG2, Populus alba L.) 0746P-0003 and -0004. 2007-3-22 
Japan Biosafety Clearing-House (J-BCH) (2008) Eucalyptus tree containing salt tolerance inducing gene $\operatorname{cod} A$ derived from Arthrobacter globformis (codA, Eucalyptus globulus Labill.) (1071, 1-9-1 and 2-1-1) 08-26P-0001, -0002, and -0003. 2008-2-8

Japan Biosafety Clearing-House (J-BCH) (2011) Eucalyptus tree containing cold tolerance inducing gene des 9 derived from Cyanobacteria (des9, Eucalyptus globulus Labill.) 11-26P-0001. 2011-6-20

Kikuchi A, Kawaoka A, Shimazaki T, Yu X, Ebinuma H, Watanabe KN (2006) Trait stability and environmental biosafety assessments on three transgenic Eucalyptus lines (Eucalyptus camldulensis Dehnh. codA 12-5B, codA 12-5C, codA 20-C) conferring salt tolerance. Breed Res 8: 17-26 (in Japanese)

Kikuchi A, Watanabe KN, Tanaka Y, Kamada H (2008) Recent progress on environmental biosafety assessment of genetically modified trees and floricultural plants in Japan. Plant Biotechnol 25: 9-16

Kikuchi A, Yu X, Shimazaki T, Kawaoka A, Ebinuma H, Watanabe KN (2009) Allelopathy assessments for the environmental biosafety of the salt-tolerant transgenic Eucalyptus camaldulensis, genotypes codA 12-5B, codA 12-5C, and codA 20C. J Wood Sci 55: 149-153

Lelmen KE, Yu X, Kikuchi A, Shimazaki T, Mimura M, Watanabe KN (2010) Mycorrhizal colonization of transgenic Eucalyptus camaldulensis carrying the mangrin gene for salt tolerance. Plant Biotechnol 27: 339-344
Khan MA, Iqtidar H, Khan EA (2008) Allelopathc effects of Eucalyptus (Eucalyptus camaldulensis L.) on germination and seedling growth of wheat (Triticum aestivum L.). PakJ Weed Sci Res 14: 9-18

Schomberg R (1998) An appraisal of the working in practice of directive 90/220/EEC on the deliberate release of genetically modified organisms. Scientific and Technological Options Assessment of the European Parliament. http://www.psrast.org/ eudircom.htm.

Shiomi M, Asakawa Y, Fukumoto F, Hamaya E, Hasebe A, Ichikawa H, Matsuda I, Muramatsu T, Okada M, Sato M, et al. (1992) Evaluation of the impact of the release of transgenic tomato plants with TMV resistance on the environment. Bull Natl Inst Agro-Environ Sci Jpn (ISSN 0911-9450) 8: 1-51 (in Japanese with English summary)

Yu X, Kikuchi A, Matsunaga E, Morishita Y, Nanto K, Sakurai N, Suzuki H, Shibata D, Shimada T, Watanabe KN (2009) Establishment of the evaluation system of salt tolerance on transgenic woody plants in the special netted-house. Plant Biotechnol 26: 135-141

Yu X, Kikuchi A, Shimazaki T, Yamada A, Ozeki Y, Matsunaga E, Ebinuma H, Watanabe KN (2013) Assessment of the salt tolerance and environmental biosafety of Eucalyptus camaldulensis harboring a mangrin transgene. J Plant Res 126: $141-150$ 